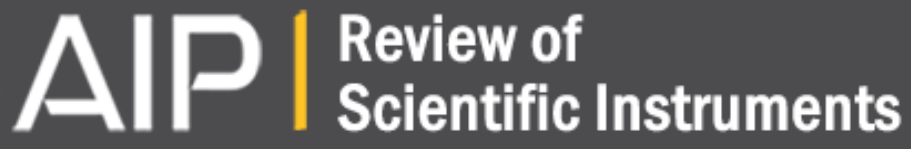

Investigation and optimization of low-frequency noise performance in readout electronics of dc superconducting quantum interference device

Jing Zhao, Yi Zhang, Yong-Ho Lee, and Hans-Joachim Krause

Citation: Review of Scientific Instruments 85, 054707 (2014); doi: 10.1063/1.4878342

View online: http://dx.doi.org/10.1063/1.4878342

View Table of Contents: http://scitation.aip.org/content/aip/journal/rsi/85/5?ver=pdfcov

Published by the AIP Publishing

\section{Articles you may be interested in}

Noise performance of lumped element direct current superconducting quantum interference device amplifiers in the $4-8 \mathrm{GHz}$ range

Appl. Phys. Lett. 97, 142502 (2010); 10.1063/1.3497008

High slew rate, ultrastable direct-coupled readout for dc superconducting quantum interference devices

Appl. Phys. Lett. 89, 063502 (2006); 10.1063/1.2335630

High frequency ac bias for direct-coupled dc superconducting quantum interference device readout electronics

Rev. Sci. Instrum. 74, 5189 (2003); 10.1063/1.1622982

Low-noise front-end electronics for solid-state fluorometers

Rev. Sci. Instrum. 70, 3759 (1999); 10.1063/1.1149989

Effect of an input coil microwave resonance on dynamics and noise properties of a dc superconducting quantum interference device operating close to the hysteretic mode

Rev. Sci. Instrum. 70, 1713 (1999); 10.1063/1.1149656

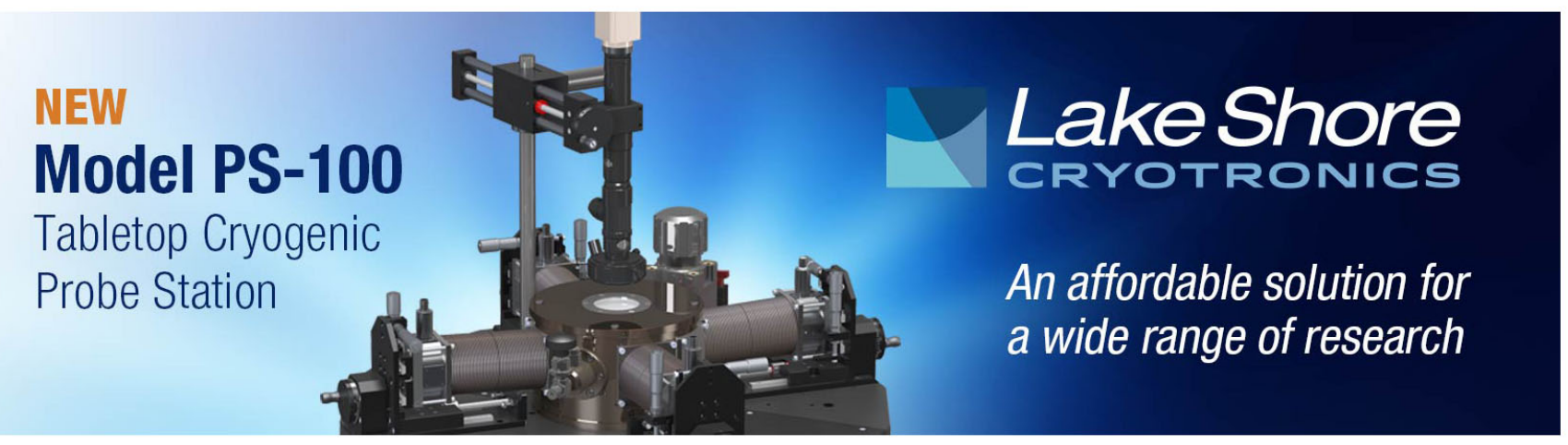




\title{
Investigation and optimization of low-frequency noise performance in readout electronics of dc superconducting quantum interference device
}

\author{
Jing Zhao, ${ }^{1,2}$ Yi Zhang, ${ }^{2}$ Yong-Ho Lee, ${ }^{2,3, a)}$ and Hans-Joachim Krause ${ }^{2}$ \\ ${ }^{1}$ College of Instrumentation and Electrical Engineering, Jilin University, Changchun 130061, China \\ ${ }^{2}$ Peter Grünberg Institute (PGI-8), Forschungszentrum Jülich (FZJ), D-52425 Jülich, Germany \\ ${ }^{3}$ Korea Research Institute of Standards and Science, Daejeon 305-340, South Korea
}

(Received 16 April 2014; accepted 6 May 2014; published online 23 May 2014)

\begin{abstract}
We investigated and optimized the low-frequency noise characteristics of a preamplifier used for readout of direct current superconducting quantum interference devices (SQUIDs). When the SQUID output was detected directly using a room-temperature low-voltage-noise preamplifier, the lowfrequency noise of a SQUID system was found to be dominated by the input current noise of the preamplifiers in case of a large dynamic resistance of the SQUID. To reduce the current noise of the preamplifier in the low-frequency range, we investigated the dependence of total preamplifier noise on the collector current and source resistance. When the collector current was decreased from $8.4 \mathrm{~mA}$ to $3 \mathrm{~mA}$ in the preamplifier made of 3 parallel SSM2220 transistor pairs, the low-frequency total voltage noise of the preamplifier (at $0.1 \mathrm{~Hz}$ ) decreased by about 3 times for a source resistance of $30 \Omega$ whereas the white noise level remained nearly unchanged. Since the relative contribution of preamplifier's input voltage and current noise is different depending on the dynamic resistance or flux-to-voltage transfer of the SQUID, the results showed that the total noise of a SQUID system at low-frequency range can be improved significantly by optimizing the preamplifier circuit parameters, mainly the collector current in case of low-noise bipolar transistor pairs. () 2014 AIP Publishing LLC. [http://dx.doi.org/10.1063/1.4878342]
\end{abstract}

\section{INTRODUCTION}

A dc SQUID (superconducting quantum interference device) system consists of a SQUID sensor and its readout electronics. The system sensitivity depends on both the SQUID intrinsic noise $\Phi_{i n}$ and the preamplifier noise $\Phi_{\text {preamp }}$. To minimize $\Phi_{\text {preamp }}$, different readout schemes were introduced. To operate a SQUID using a direct readout scheme (DRS), reduction of preamplifier noise contribution is always required. There are two different ways for this: (1) to increase the fluxto-voltage transfer coefficient $\partial V / \partial \Phi$ of the SQUID, e.g., using a voltage feedback circuit, such as additional positive feedback (APF), ${ }^{1}$ noise cancellation (NC), ${ }^{2}$ SQUID bootstrap circuit $(\mathrm{SBC}){ }^{3}$ double relaxation oscillation SQUID,${ }^{4}$ $\mathrm{dc}$ SQUID with small-sized junctions, ${ }^{5}$ or a weakly damped SQUID, ${ }^{6}$ and (2) to reduce the preamplifier noise directly.

An operational amplifier (e.g., AD797 (Analog Device)) is usually employed as a preamplifier in DRS. The preamplifier noise consists of two components, voltage noise $V_{\mathrm{n}}$ and current noise $I_{\mathrm{n}}$. Typically, the voltage noise $V_{\mathrm{n}}$ of about $1 \mathrm{nV} / \sqrt{ } \mathrm{Hz}$ dominates the preamplifier noise contribution while the current noise $I_{\mathrm{n}}$ of about $2 \mathrm{pA} / \sqrt{ } \mathrm{Hz}$ in the white noise region can be neglected for SQUID readout. ${ }^{7}$

To improve the noise impedance match between SQUID and preamplifier, several pairs of bipolar-type transistors (e.g., SSM2210 or SSM2220) are connected in parallel to reduce the voltage noise $V_{\mathrm{n}}$ of a single transistor to $V_{\mathrm{n}} / \sqrt{ } n$ ( $n$ : number of transistors). We call such type of preamplifier a parallel-

a) Author to whom correspondence should be addressed. Electronic mail: yhlee@kriss.re.kr connected bipolar transistor (PCBT). At $n=6$, the voltage noise of the PCBT can be reduced to about $0.35 \mathrm{nV} / \sqrt{ } \mathrm{Hz}$ in the white noise frequency region. ${ }^{8}{ }^{8}$ In this case, the current noise of PCBT increases, however, to $\sqrt{ } n \times I_{\mathrm{n}}$, so that its contribution should not be neglected.

In this study, we investigate the noise performance of PCBT at $n=6$ with different collector currents to find the optimal noise impedance match. The SQUIDs with different Stewart-McCumber parameters $\beta_{\mathrm{c}}$ exhibit different dynamic resistances $R_{\mathrm{d}}$. It is found that the current noise of PCBT dominates the SQUID system noise in the low frequency region in the case of a large $R_{\mathrm{d}}$. Indeed, the collector current of PCBT is a key parameter to balance both $V_{\mathrm{n}}$ and $I_{\mathrm{n}}$ for different $R_{\mathrm{d}}$ (or $\beta_{\mathrm{c}}$ ), thus minimizing the total preamplifier noise contribution of the PCBT.

\section{LOW-NOISE PREAMPLIFIER}

\section{A. Preamplifier circuit}

Figure 1(a) schematically describes a PCBT preamplifier consisting of three pairs of SSM2220 (audio dual matched PNP transistors), i.e., $n=6$. Because the base voltages $V_{\mathrm{b}+}$ and $V_{\mathrm{b}-}$ approach zero, the emitter voltage $V_{\mathrm{e}}$ remains at $0.6 \mathrm{~V}$. The total collector current $I_{\text {col }}$ of the 6 transistors is controlled by an adjustable current source located below the transistor emitters. We hold the collector voltage $V_{\mathrm{c}} \approx-1 \mathrm{~V}$ by adjusting $V_{-}$. Here, two high-precision collector resistors $R_{c o l}=1.5 \mathrm{k} \Omega(0.05 \%)$ were used for the symmetric balance of this differential circuit. At $V_{\mathrm{b}+}$, we introduce a resistor $R_{\mathrm{s}}$, which simulates the SQUID dynamic resistance $R_{\mathrm{d}}$, to perform the noise measurements. Indeed, the circuit of Fig. 1(a) 


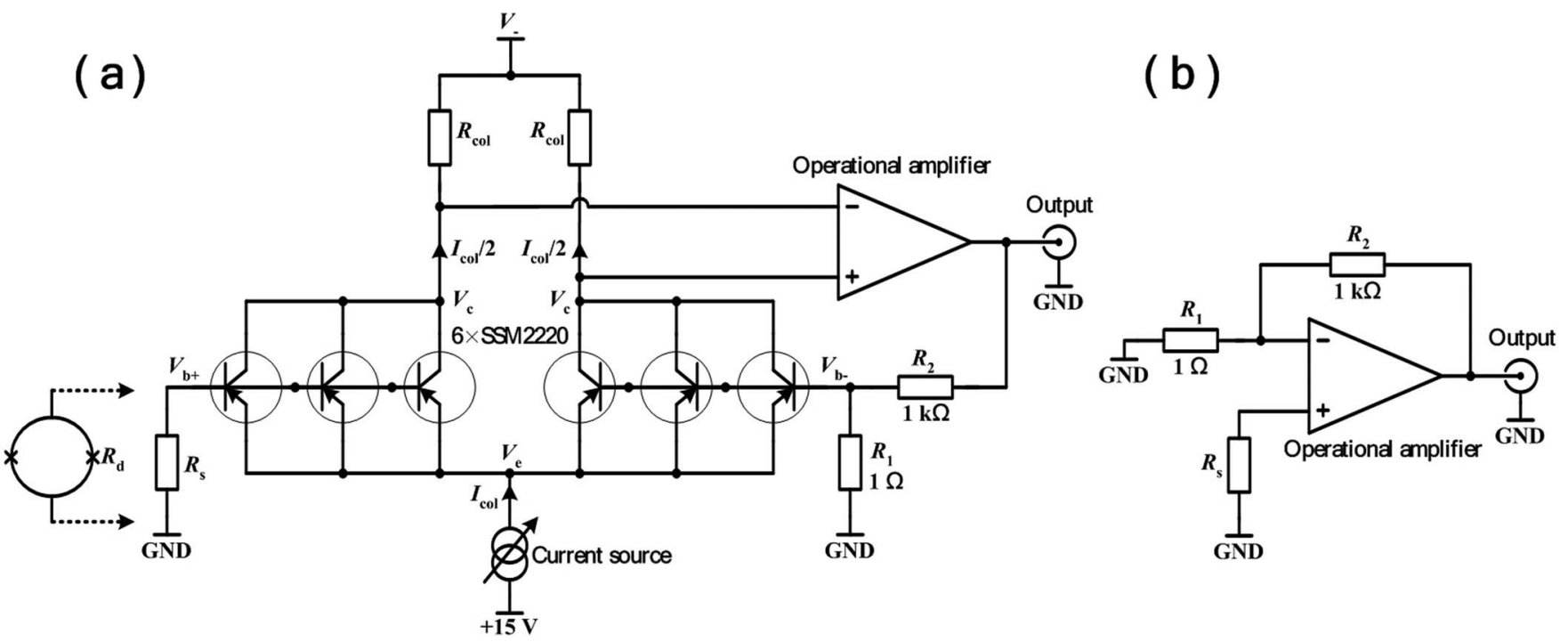

FIG. 1. Circuit diagram of the preamplifier of PCBT consisting of three pairs of SSM2220. (a) Details of the circuit diagram, and (b) equivalent functional diagram of the preamplifier as an operational amplifier.

functions as an operational amplifier as shown in Fig. 1(b), whose gain $G(=1000)$ is determined by $R_{2} / R_{1}$.

\section{B. Preamplifier input noise vs. collector current}

The preamplifier input total voltage noise $V_{\mathrm{n} \text {,tot }}$ consists of 3 components, i.e., the input voltage noise $V_{\mathrm{n}}$, the input current noise $I_{\mathrm{n}}$, and the Nyquist noise $T_{\mathrm{n}}=\sqrt{ } 4 k_{\mathrm{B}} T R_{\mathrm{S}}$ of the source resistance $R_{\mathrm{s}}$. The relation between them is

$$
V_{n, t o t}=\left(V_{n}^{2}+\left(I_{n} R_{s}\right)^{2}+4 k_{B} T R_{S}\right)^{0.5},
$$

where $k_{\mathrm{B}}$ is the Boltzmann constant and $T$ denotes the environmental temperature at $R_{\mathrm{s}}$. It is found that both $V_{\mathrm{n}}$ and $I_{\mathrm{n}}$ vary with collector currents $I_{\text {col }}$. By changing $I_{\text {col }}$ and $R_{\mathrm{s}}$, we measured $V_{\mathrm{n}}, I_{\mathrm{n}}$, and $V_{\mathrm{n} \text {,tot }}$ with a dynamic signal analyzer (HP35670A). Flat top window was used for the averaging. Due to limitation in the frequency resolution of HP35670A, the spectral curves were taken in 3 different frequency ranges and then combined together to have a single wide window range.

By shorting the preamplifier input, the voltage noise $V_{\mathrm{n}}$ was measured for 3 different values of the collector current $I_{\text {col }}(0.3,3.0$, and $8.4 \mathrm{~mA})$. The result is shown in Fig. 2. The corresponding voltage noise values $V_{\mathrm{n}}$ are $1.43,0.46$, and $0.34 \mathrm{nV} / \sqrt{ } \mathrm{Hz}$ at $1 \mathrm{kHz}$, for $I_{\text {col }}$ of $0.3,3.0$, and $8.4 \mathrm{~mA}$, respectively. The frequency dependence of $V_{\mathrm{n}}$ is similar, with the $1 / f$ corner frequency around $1 \mathrm{~Hz}$ for all the 3 cases. At $0.1 \mathrm{~Hz}, V_{\mathrm{n}}$ values of $3.66,1.15$, and $1.64 \mathrm{nV} / \sqrt{ } \mathrm{Hz}$ were found for $I_{\mathrm{col}}$ of $0.3,3.0$, and $8.4 \mathrm{~mA}$, respectively. Comparing the two $V_{\mathrm{n}}$ values for $I_{\text {col }}$ of $3.0 \mathrm{~mA}$ and $8.4 \mathrm{~mA}$, it is clear that $V_{\mathrm{n}}$ is higher for lower $I_{\mathrm{col}}$ in white noise region while lower in the low frequency region (below $1 \mathrm{~Hz}$ ), so that preamplifiers exhibiting lower white voltage noise do not necessarily yield lower $1 / f$ noise. Particularly, if $I_{\text {col }}$ is too low (e.g., $0.3 \mathrm{~mA}), V_{\mathrm{n}}$ becomes much higher in the whole frequency range measured.

To measure $I_{\mathrm{n}}$, a $50-\Omega$ resistor was used as source resistance. To minimize the influence of Nyquist noise, and thus the error in measuring $I_{\mathrm{n}}$, the resistor was cooled in liquid Helium using a SQUID insert. For connection to room temperature, twisted $\mathrm{Cu}$ wire with a total resistance of $1.5 \Omega$ was used. From the measured total voltage noise, the current noise was calculated using the relation $I_{\mathrm{n}}=\left(V_{\mathrm{n}, \text { tot }}^{2}-V_{\mathrm{n}}^{2}-4 k_{\mathrm{B}} T R_{\mathrm{s}}-4 k_{\mathrm{B}} T_{\mathrm{av}} R_{\mathrm{w}}\right)^{0.5} / R_{\mathrm{s}}$, where $T_{\mathrm{av}}$ denotes the average temperature of the $\mathrm{Cu}$ wire (assumed to be $150 \mathrm{~K}$ ), and $R_{\mathrm{W}}$ is the average resistance of the $\mathrm{Cu}$ wire.

The current noise $I_{\mathrm{n}}$ in the white frequency region at $10 \mathrm{kHz}$ was found to be $13.45,6.90$, and $7.23 \mathrm{pA} / \sqrt{ } \mathrm{Hz}$ at collector currents $I_{\text {col }}$ of $0.3,3.0$, and $8.4 \mathrm{~mA}$, respectively (shown in Fig. 3). Though differences in the current noise $I_{\mathrm{n}}$ for different values of the collector currents $I_{\text {col }}$ are not large in the white noise region, the current noise becomes larger at lower frequencies (below about $10 \mathrm{~Hz}$ ). For the highest $I_{\text {col }}$ (i.e., $8.4 \mathrm{~mA}$ ), it starts to increase already from about $1 \mathrm{kHz}$, with a $1 / f$ corner frequency of about $400 \mathrm{~Hz}$. By decreasing the collector current, the curves become more flattened

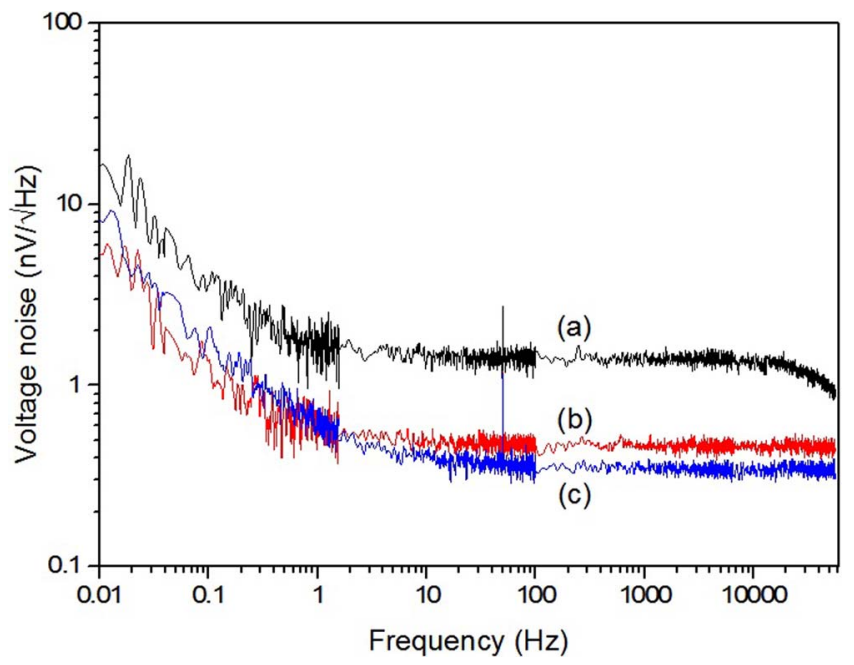

FIG. 2. Input voltage noises $V_{\mathrm{n}}$ of the preamplifier with shorted input, for 3 different collector currents $I_{\text {col }}$ : (a) 0.3 , (b) 3.0 , and (c) $8.4 \mathrm{~mA}$, respectively. 


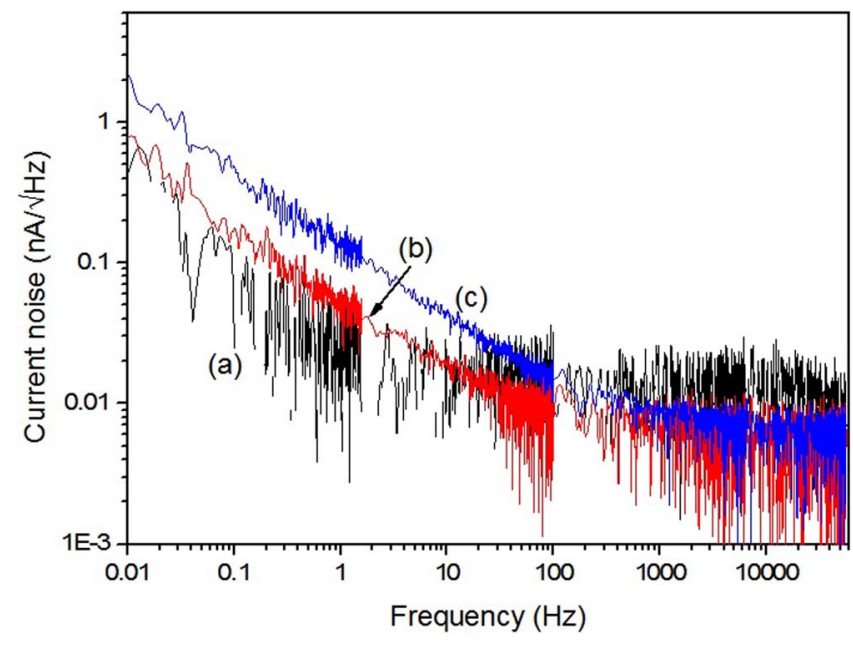

FIG. 3. Input current noises $I_{\mathrm{n}}$ of the preamplifier for 3 different collector currents $I_{\mathrm{col}}$ : (a) 0.3 , (b) 3.0 , and (c) $8.4 \mathrm{~mA}$, respectively.

toward the lower frequency region and the $1 / f$ corner frequency decreases.

We changed the source resistance stepwise and compared preamplifier input total noise $V_{\mathrm{n}, \text { tot }}$ depending on $I_{\mathrm{col}}$. Among them, Fig. 4 shows the $V_{\mathrm{n}, \text { tot }}$ for $R_{\mathrm{s}}$ of $30 \Omega$ at 3 different $I_{\text {col }}$. Here, the resistor was also cooled in liquid Helium. At the lowest $I_{\mathrm{col}}$ of $0.3 \mathrm{~mA}, V_{\mathrm{n}, \text { tot }}$ is highest in the white noise region, but it is lowest for low frequencies (below $1 \mathrm{~Hz}$ ). At $I_{\text {col }}$ $=3.0 \mathrm{~mA}$, low-frequency noise is a little higher while white noise is much smaller than those of $I_{\mathrm{col}}=0.3 \mathrm{~mA}$. On the other hand, the largest $I_{\mathrm{col}}$ of $8.4 \mathrm{~mA}$ yielded the lowest white noise, but the low-frequency noise is much higher than those of lower $I_{\mathrm{col}}$ values. The increase of low-frequency noise at the highest $I_{\mathrm{col}}$ value is definitely caused by the larger $I_{\mathrm{n}}$ at low frequencies. For a source resistance of $30 \Omega$ at a frequency of $0.1 \mathrm{~Hz}$, the current noise contribution dominates the total voltage noise.

\section{Preamplifier input noise vs. source resistance}

From Fig. 4, we can see that the noise curves cross each other at certain frequencies, caused by different contribution

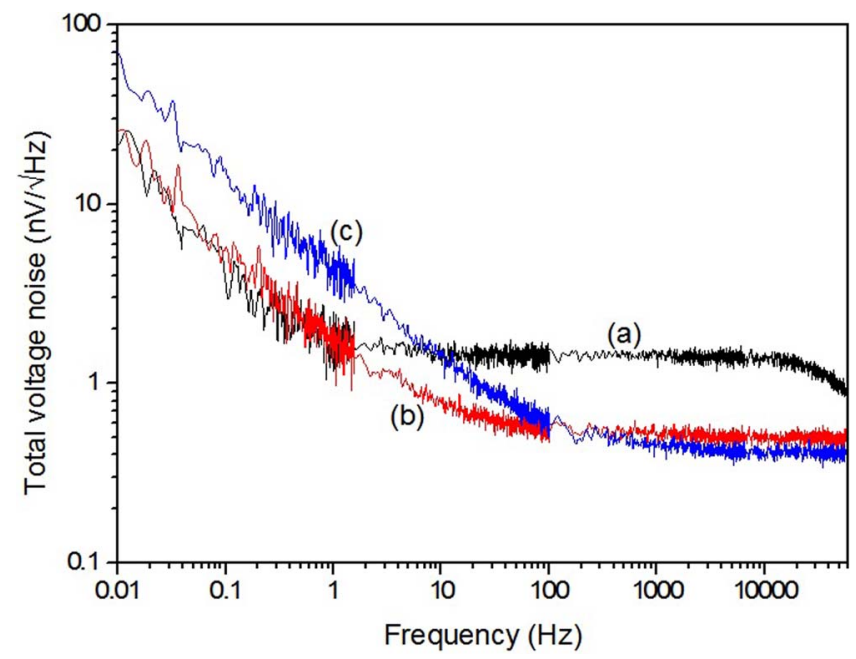

FIG. 4. Total voltage noise $V_{\mathrm{n}, \text { tot }}$ for a source resistance of $30 \Omega$ at 3 different collector currents $I_{\mathrm{col}}$ : (a) 0.3 , (b) 3.0 , and (c) $8.4 \mathrm{~mA}$, respectively.

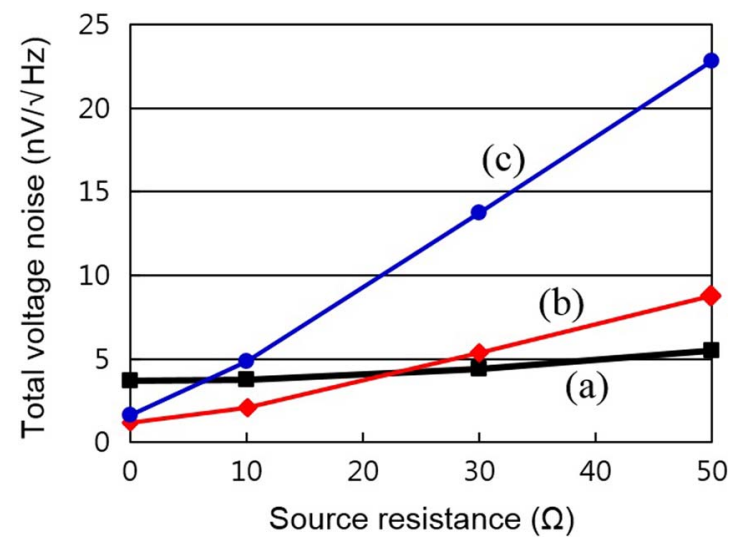

FIG. 5. Total voltage noise $V_{\mathrm{n} \text {,tot }}$ vs. source resistance $R_{\mathrm{S}}$ for 3 collector currents at $0.1 \mathrm{~Hz}$ : (a) $I_{\text {col }}$ of 0.3 , (b) 3.0 , and (c) $8.4 \mathrm{~mA}$, respectively.

of voltage noise $V_{\mathrm{n}}$ and current noise $I_{\mathrm{n}} R_{\mathrm{s}}$ at each frequency. We compared the behavior of $V_{\mathrm{n} \text {,tot }}$ at $0.1 \mathrm{~Hz}$ depending on the $R_{\mathrm{S}}$, and showed the results in Fig. 5. As expected, the lower collector currents (Figs. 5(a) and 5(b)) having lower current noise exhibit a slow increase of $V_{\mathrm{n}, \text { tot }}$ with increasing source resistance. But, in case of $I_{\mathrm{col}}=8.4 \mathrm{~mA}, V_{\mathrm{n} \text {,tot }}$ increases rapidly with the source resistance.

For each frequency, we can define a threshold resistance as the ratio of voltage noise to current noise, $R_{\mathrm{th}}=V_{\mathrm{n}} / I_{\mathrm{n}}$. At $0.1 \mathrm{~Hz}$, the threshold resistance $R_{\text {th }}$ is $44.4,6.6$, and $3.6 \Omega$ for collector currents $I_{\text {col }}$ of $0.3,3.0$, and $8.4 \mathrm{~mA}$, respectively. Thus, if $R_{\mathrm{s}}$ is larger than $R_{\mathrm{th}}$ of that frequency, the current noise contribution becomes dominant for frequencies below this frequency, so that reduction of current noise is needed by reducing the collector current. In the white noise region at $10 \mathrm{kHz}$, the threshold values $R_{\mathrm{th}}$ are $102.1,66.7$, and $40.5 \Omega$, for $I_{\text {col }}$ of $0.3,3.0$, and $8.4 \mathrm{~mA}$, respectively. Thus, the contribution of the current noise $I_{\mathrm{n}}$ is smaller than the voltage noise $V_{\mathrm{n}}$ if the SQUID dynamic resistance is smaller than $40.5 \Omega$, which is usually the case for dc SQUIDs having $\partial V / \partial \Phi$ less than about $500 \mu \mathrm{V} / \Phi_{0}$. For higher values of $\partial V / \partial \Phi$ or higher $R_{\mathrm{d}}$, the contribution of $I_{\mathrm{n}}$ can be dominant.

\section{SQUID NOISE MEASUREMENT}

\section{A. SQUID parameters}

We tested the SQUID flux noise using a dc SQUID with a Ketchen-type structure with two square holes connected in parallel to form a gradiometer configuration. ${ }^{10}$ The SQUIDs were fabricated using standard $\mathrm{Nb} / \mathrm{AlO}_{\mathrm{x}} / \mathrm{Nb}$ Josephson junction technology. The size of each junction is $3 \mu \mathrm{m} \times 3 \mu \mathrm{m}$. The SQUID was shielded using a superconducting $\mathrm{Nb}$ tube and immersed in a stainless-steel liquid Helium container without additional magnetic shielding. The SQUID inductance $L$ is $125 \mathrm{pH}$, and the maximum critical current is 12.5 $\mu \mathrm{A}$. From the current-voltage curve of the SQUID, the dynamic resistance $R_{\mathrm{d}}$ was measured to be $33 \Omega$, which is much larger than the shunt resistance per junction $R_{\mathrm{J}}$ of $6.1 \Omega$. The flux-to-voltage characteristics of the SQUID is shown in Fig. 6 . The modulation curve is quite steep with a swing voltage of $46 \mu \mathrm{V}_{\mathrm{pp}}$ and $\partial V / \partial \Phi$ of $410 \mu \mathrm{V} / \Phi_{0}$. 


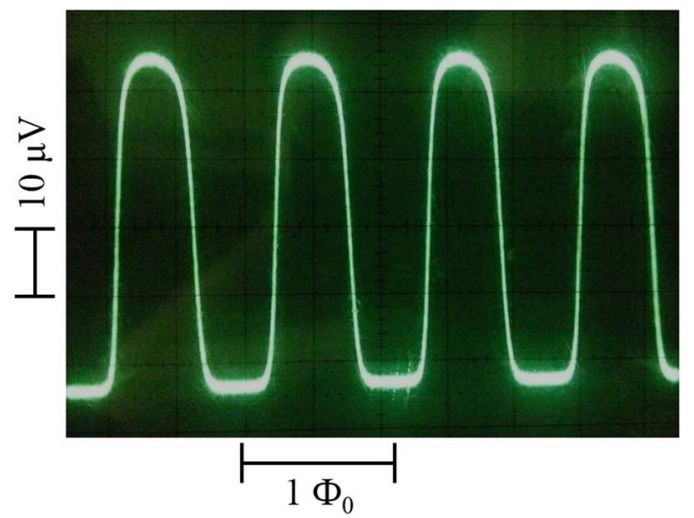

FIG. 6. A flux-voltage curve of the dc SQUID.

\section{B. SQUID noise}

Flux noise spectra of the SQUID system at 3 different $I_{\mathrm{col}}$ are shown in Fig. 7. Even though the intrinsic noise of the SQUID is the same, the noise of the SQUID system shows different patterns depending on $I_{\mathrm{col}}$. In other words, different noise characteristics of the preamplifier become dominant at different collector currents. Since the dynamic resistance of the SQUID is $33 \Omega$, the SQUID flux noise curves of Fig. 7 are quite similar to the noise curves of Fig. 4 corresponding to a source resistance $R_{\mathrm{s}}$ of $30 \Omega$. This means that it is possible to predict the low-frequency noise behavior of a SQUID system if we know the dynamic resistance of the SQUID and its intrinsic flux noise. And, SQUIDs of similar dynamic resistances yield similar flux noise curves.

In Fig. 8, we decomposed the measured SQUID system noise into preamplifier noise contribution and SQUID intrinsic noise. The SQUID noise measurement was done with $I_{\mathrm{col}}$ of $3.0 \mathrm{~mA}$, corresponding to the curve of Fig. 7(b). The preamplifier noise contribution was calculated from $V_{\mathrm{n} \text {,tot }}$ of the preamplifier for a source resistance of $33 \Omega$, divided by $410 \mu \mathrm{V} / \Phi_{0}$. The intrinsic SQUID noise exhibits a white noise level of $1.2 \mu \Phi_{0} / \sqrt{ } \mathrm{Hz}$ and a typical $1 / f^{\alpha}$ behavior for decreasing frequency, with $\alpha=0.5$ and a $1 / f$ corner frequency of

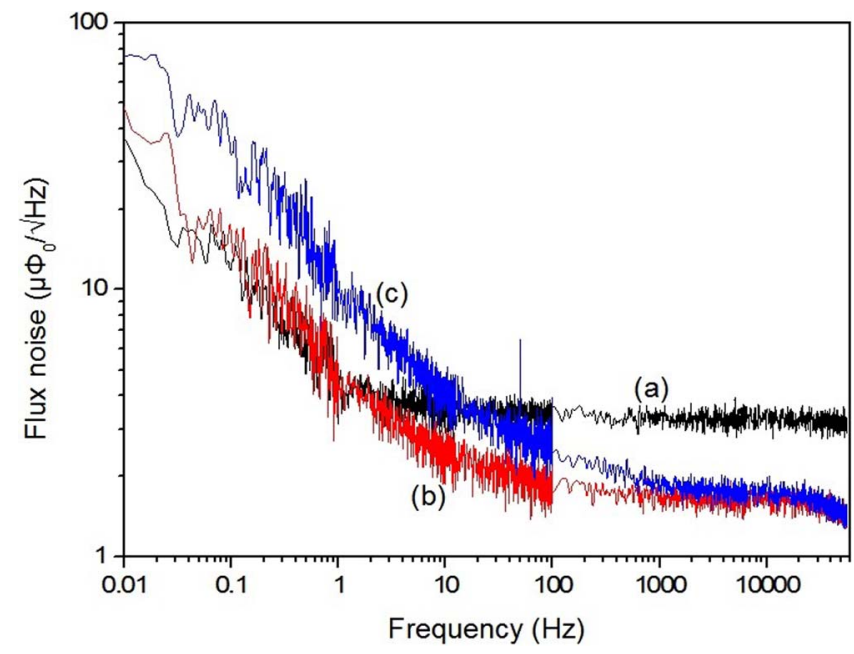

FIG. 7. Noise spectra of the SQUID system with different collector currents $I_{\mathrm{col}}$ : (a) 0.3 , (b) 3.0 , and (c) $8.4 \mathrm{~mA}$, respectively.

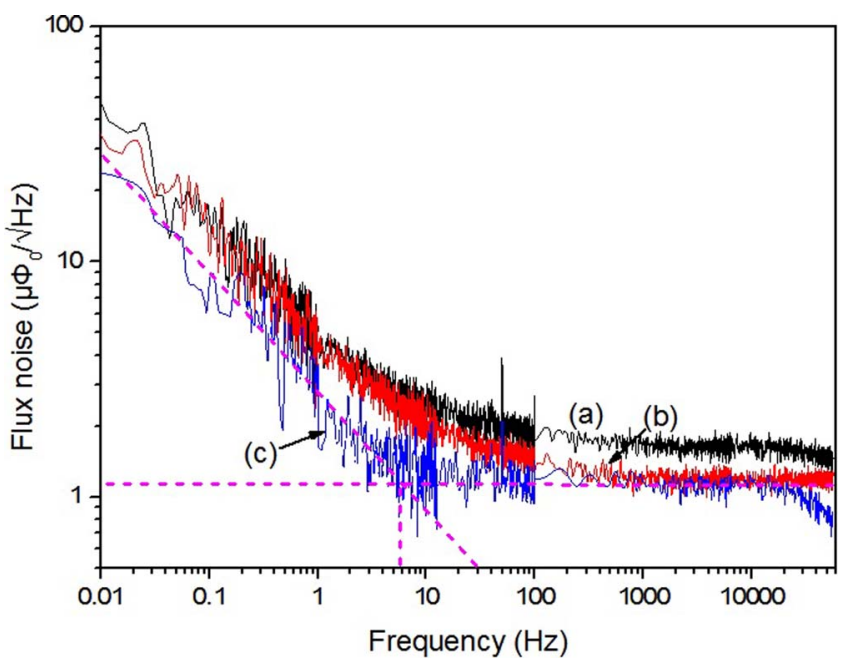

FIG. 8. Sources of noise. (a) Total noise of the SQUID system in the FLL operation, (b) preamplifier noise contribution, and (c) intrinsic noise of the SQUID. The collector current was set to $3.0 \mathrm{~mA}$.

$6 \mathrm{~Hz}$. Using the equation for the intrinsic noise of the uncoupled SQUID, ${ }^{11} \Phi_{n}=\left[2 k_{B} T\left\{L^{2} / R_{J}+2 R_{d} / V_{\Phi}{ }^{2}\right\}\right]^{0.5}$, the theoretical intrinsic noise is $0.34 \mu \Phi_{0} / \sqrt{ } \mathrm{Hz}$. Thus, the measured intrinsic noise level is only about 3.5 times larger than the theoretical one.

Even though the total voltage noise of the preamplifier was optimized by adjusting the collector current, the preamplifier still contributes most part of the system noise in the low-frequency range as shown in Fig. 8. This shows that it is indispensable to consider the preamplifier input noise when optimizing direct SQUID readout. If one used a preamplifier having a higher input noise, the total system noise would have increased accordingly, especially in the low-frequency range.

\section{CONCLUSION}

Conventional approach considering only voltage noise with a shorted input does not result in the lowest total noise of a SQUID system. As the source resistance of the preamplifier increases, the contribution of current noise increases and becomes dominant above a threshold resistance. Depending on the dynamic resistance of the SQUID, the contribution of the current noise can be larger than the voltage noise contribution, so that dynamic resistance should be considered in preamplifier design. All the approaches to increase the fluxto-voltage transfer of the SQUID, such as additional positive feedback, ${ }^{1}$ noise cancellation, ${ }^{2}$ SQUID bootstrap circuit, ${ }^{3}$ double relaxation oscillation SQUID, ${ }^{4}$ dc SQUID with smallsized junctions, ${ }^{5}$ or a weakly damped SQUID,${ }^{6}$ all reduce the contribution of preamplifier noise, but inevitably increase the dynamic resistance. Thus, the contribution of the current noise should be considered for low-noise SQUID systems especially when using these SQUID configurations. By optimizing the collector current of the preamplifier, we found a setting that yielded the lowest total noise in the low-frequency range. For a preamplifier made of 3 SSM2220 transistor pairs and for a SQUID dynamic resistance of $33 \Omega$, a collector current of $3.0 \mathrm{~mA}$ resulted in the lowest total noise in both the white 
noise and the low-frequency regime. Another advantage of a lower collect current is lower power consumption, resulting in reduced voltage drift. Avoiding such drift is a serious issue in multichannel SQUID systems.

${ }^{1}$ D. Drung, R. Cantor, M. Peters, H. J. Scheer, and H. Koch, Appl. Phys. Lett. 57, 406 (1990).

${ }^{2}$ M. Kiviranta and H. Seppä, IEEE Trans. Appl. Supercond. 5, 2146 (1995).

${ }^{3}$ X. Xie, Y. Zhang, H. Wang, Y. Wang, M. Mück, H. Dong, H. J. Krause, A. I. Braginski, A. Offenhäusser, and M. Jiang, Supercond. Sci. Technol. 23, 065016 (2010).

${ }^{4}$ Y. H. Lee, H. Kwon, J. M. Kim, Y. K. Park, and J. C. Park, Supercond. Sci. Technol. 12, 943 (1999).
${ }^{5}$ M. Schmelz, R. Stolz, V. Zakosarenko, T. Schönau, S. Anders, L. Fritzsch, M. Mück, and H.-G. Meyer, Supercond. Sci. Technol. 24, 065009 (2011).

${ }^{6}$ J. Zeng, Y. Zhang, M. Mück, H. J. Krause, A. I. Braginski, X. Kong, X. Xie, A. Offenhäusser, and M. Jiang, Appl. Phys. Lett. 103, 122605 (2013).

${ }^{7}$ G. Zhang, Y. Zhang, H. Dong, H.-J. Krause, X. Xie, A. I. Braginski, A. Offenhäusser, and M. Jiang, Supercond. Sci. Technol. 24, 065023 (2011).

${ }^{8}$ N. Oukhanski, R. Stolz, V. Zakosarenko, and H.-G. Meyer, Physica C 368, 166 (2002).

${ }^{9}$ J. Zeng, Y. Zhang, M. Mück, H. J. Krause, A. I. Braginski, X. Kong, X. Xie, A. Offenhäusser, and M. Jiang, Appl. Phys. Lett. 103, 042601 (2013). ${ }^{10}$ M. B. Ketchen, J. Appl. Phys. 58, 4322 (1985).

${ }^{11} \mathrm{H}$. Weinstock, in SQUID Sensors: Fundamentals, Fabrication and Applications, edited by R. Cantor (Kluwer Academic Publishers, Dordrecht, 1996), p. 179. 